\title{
Designing a Rule Based Expert Systems for Contact Lenses Patients
}

\author{
İbrahim Berkan Aydilek \\ Harran University Computer Engineering, Şanlıurfa, 63300, Turkey \\ E-mail: berkanaydilek@harran.edu.tr \\ Abdülkadir Gümüşçü \\ Harran University Electrical-Electronics Engineering, Şanlıurfa, 63300, Turkey \\ E-mail: agumuscu@harran.edu.tr
}

Received: 04 November 2017; Accepted: 07 December 2017; Published: 08 March 2018

\begin{abstract}
Expert systems that bring facts and valuable experiences together and make some deductions possible. Expression of relevant knowledge and experience in these structures may be in a set of rules. Learning problems are valid with expert systems. Therefore, they cannot add new rules and information automatically by themselves. Rules are created by human experts on the way and added upon the system. Classification datasets are collections of data commonly used in machine learning that contain and classify the previously obtained experiences. In this study, rules were obtained by using Part, NNge, Prism rule classifier algorithms, and a knowledge base of expert systems was systematically created to achieve enrichment Enrichment and rule deduction process needs careful and sensitive attention. A combined methodology and study was revealed during this sensitive process. In this context, studies were conducted on five widely used datasets. It was aimed to reduce the redundant, conflicting, subsumed and circular rules in order to create a consistent and complete knowledge base. In this way, a methodology was developed to establish more powerful and richer contents of knowledge base that have higher quality.
\end{abstract}

Index Terms-Knowledge acquisition, Rule extraction, Expert systems, Lenses Dataset, Rule Checker Algorithms.

\section{INTRODUCTION}

At present, the existence of various applications of expert systems in different fields of knowledge is quite common [1], [2]. It has become quite essential to develop the information technology, decision making systems, or the entire decision making based on computer. Regarding this, there is a primary role of expert systems involving ascribing of parts to artificial intelligence. In an expert system, all types of decisions are considered through the computer. Expert systems are like the basis of knowledge, or they are in reality, the most significant part of them. In these systems, knowledge is transferred from expertise of any field to the computer [3], [4], [5]. An expert system is a type of information system and performs like a human expert for users by applying the knowledge base rules in the specific field. An expert system includes a knowledge base and software module with an inference engine for decision support so that end users could have some advice regarding the decision. Data mining which combines methods like machine learning, artificial intelligence, database systems, statistics, and pattern recognition is utilized for analysis of high volumes of data. Many data-mining algorithms are available for data analysis of different tasks [6]. Expert systems are also developed through data mining approaches. Knowledge, in a rule-based knowledge approach, is represented in terms of condition statements, like it-then rules. There are a number of applications of rule-based expert systems [7], [8]. Expert systems come under applied artificial intelligence. They were developed in the mid of 1960s by the artificial intelligence community [7], [9]. In an expert system, the main aspect is its expertise, which is the immense amount of task-specific knowledge, is transferred to a computer through a human. This knowledge is saved in the computer and is used by users through the computer whenever required for advice. The computer makes inferences and reaches a certain conclusion. Similar to a human consultant, the computer then presents advices and it even presents explanations for the given advice, if required. An expert system is a strong and flexible way for attaining solutions to different problems which are not usually addressed through traditional approaches. For this reason, the use of expert systems is increasing and also being used in a number of sectors of our social and technological life. In fact, in those the use of expert systems is becoming quite a significant aspect during problem solving and decision support [9-18].

Expert systems are systems that are able to make deductions about a certain field just like an expert of that field. The issue to be considered in the design of expert systems is knowledge base, which is a block of knowledge that consists of rules. The knowledge obtained by utilizing the human expert is established in form of rules to be evaluated by the inference engine. Knowledge base has an important role in results to be obtained and in 
deductions. Classification data sets are collections of data where raw data about events, experiments or facts, or lived or encountered experiences are collected in text form. While these datasets can provide predictions and classifications towards the future, experiences that define these datasets can also be used for information purposes. Information can be expressed in form of rules in descriptions. Information that can be expressed in terms of if-then statements is usually sufficient to make logical deductions. Inference of rules from the classification data and usage of these as a knowledge base of expert systems can be achieved if the necessary attention is paid to the establishment of the knowledge base. If there is classification data in addition to the information obtained from a domain expert, it is possible to create rich and consistent knowledge bases as in methods explained in this study. It is known that rules can be obtained from decision trees and data [19]. After obtaining these rules, the rules are turned into valuable information. And this valuable information turns into utility in decision making and deduction in structures such as expert systems. It is smarter to utilize expert systems in areas where there are few or no domain experts, or at places that are dangerous to work for humans such as high radiation areas like reactor chambers in nuclear power plants. Applications where the expert is relocated or retired, and the need to finish the analysis in a shorter time period are other examples of ways expert systems are useful. Even more comprehensive deductions can be made, ensuring that the expert system makes its assessments in points of view of more than one experts by combining classification data. One disadvantage of an expert system is that it cannot learn new information or rules. That is, expert systems, unlike humans, cannot create new information in time or make new deductions outside the already learned. So, the information must be put into the knowledge base by a knowledge engineer. Additionally, expert people might sometimes not know how to express their knowledge, or they might not be willing to do so [20]. Knowledge engineers will be able to overcome problems and restrictions about subjects like not being able to learn or gather other information, by enriching the expert system rapidly and consistently via the classification data that contains raw experience and data, using the alternative approaches indicated in this study.

\section{RELATED WORKS}

Omid [19] designed an expert system to distinguish open and closed shells in nuts by converting the rules obtained from J48 decision tree output into if-then statements and fuzzy classifier membership function sets. With the open and closed shells data that contains 300 entries, a successful system evaluation was made. The disadvantage of this study was that an evaluation study and a methodology were not sufficiently presented about whether the rules were consistent and comprehensive or not.

Chang et al. [21] suggested a rule-based expert system with a classifying approach to solve classification problems. In their research, the expert system classifier had to be optimized according to domain experts and/or previous knowledge. Therefore, implementation of a smaller scale rule-based expert system that is able to make good correspondences was achieved by making new activations for rules and intense calculations. The advantage of the study was that the optimum parameter values for the expert system could be identified for the inference engine and optimization engine.

D'Haen et al. [22] developed a decision support system for the lead qualification process. They completed missing information with web crawling data in order to improve commercial information to be used in the decision support system. They conducted their research by combining the obtained data and expert knowledge. The advantage of their research was the demonstration of added utility in decision support systems with the usage of expert knowledge and added obtained knowledge.

Yin et al. [23] gathered deductions from the experiences of subway train operators as a collection of expert knowledge rules. They aimed to achieve safe operation and easy usage of train structures. They gathered valuable knowledge from past operation data with the algorithm named CART based on classification and regression trees. Expert knowledge and data mining algorithms were used together to improve subway train operations. Eventually, a method that surpasses manual operation was developed. The disadvantage of their study was that the obtained rules could not be subjected to a certain value measurement and consistency analysis, and therefore they could not be simplified.

Ho et al. [24] suggested a methodological structure that is able in fuzzy linguistic group decision-making in order to establish a successful and high quality case-based reasoning (CBR) system. They created a $\mathrm{KC}$ index that is valid based on membership function parameters labeled by domain experts and comprehensively in terms of the measurement weights of the experts. According to the results of the experiments, it was proven that such a hybrid structure is very effective in establishing a casebased knowledge system and it is a powerful methodology in terms of performance. One shortcoming of their study was that a probability-based rule combination method was not used to evaluate views taken from more than one expert.

Laasri et al. [25] used a fuzzy rule-based expert system for seismic signal classification. Their purpose was to develop a rule-based expert classification system that can work in cooperation with the analysis information of seismic case classification and imitate human evaluation and deduction processes. The main idea behind the establishment of this approach is to learn from which experimental rules human analysts are able to classify seismic cases. The information used in the study was gathered from both expert knowledge and data. A disadvantage of the study was that they did not prefer a probability-based method in conflict analysis.

Seok et al. [26] developed an automated system to determine the age of whole and partial bones. They conducted interviews with two domain experts and 
learned their strategies to determine age, and bone age determination was made automatically by deriving rules about bone age estimation and bone union. The results show that the algorithm has a considerable classification capability.

Ghanbari et al. [27] emphasized that knowledge-based expert systems have come to be important tools for scientists and engineers. They conducted a study that showed these systems have many properties to overcome real or imaginary engineering problems that cannot be solved with cognitive approaches. They established an expert system with the ability to imitate fluctuations in energy demands under factors that affect consumption and named it "Cooperative Ant Colony OptimizationGenetic Algorithm" (COR-ACO-GA). In the first stage, they created the data base of the expert system with genetic algorithm. In the second stage, they increased the success of the method by intensifying the relationship of fuzzy linguistic rules, and the data base and rule base. The proposed method found to be more accurate and consistent in comparison to the neuro-fuzzy deduction system and artificial neural network.

Murianaa et al. [28] created a model of relationships and correlations between input and output with the help of the deduction rules obtained from the data in the form of time series. They presented the created model as an expert system based on a fuzzy set engine. They focused on the financial performance analysis of a medical center such as a hospital. In this interdisciplinary type of study, they investigated which indicators used for more than one input affected financial performance and in what ways these effects worked. Output measurement calculations were made by the expert system. Then the parameters were estimated using adaptive estimation methods and the required value was compared with the obtained value. Results suggested that deduction measurements were better and adaptive models and they model input-output relationships more effectively.

Lee et al. [29] developed a rule-based shopping expert system. According to high quality estimations, they made improvements and fortifications on the rule-based system in terms of usage and performance. Using this fuzzy cognitive map, the relevant relationships and their directions and magnitude were computed in internetbased stocks purchasing planning systems.

Xavier et al. [30] designed an expert system to automatically predict gene order in the DNA. It was shown with this expert system that estimation quality increased, while the work load for experts decreased effectively. In the article, a rule-based expert system that can imitate human discernment abilities was developed. The system combined heuristic usage of bioinformatics tools and biology. The advantage of the study was that they showed the benefits of expert systems in knowledge acquisition and formation. The results gathered in the first protein function evaluation process showed great performance.

\section{RULE BASED EXPERT SYSTEMS}

Knowledge can be expressed as sum of what are known also knowledge is power. Expert is a human who have profound and wide information. Domain expert have practical experience and specific information based on facts and rules in specific domain. A human expert is person who can do something related subject rather than other persons. Human mental and reasoning process, due to too complex, is hard to describe by algorithms. Therefore, many experts state their knowledge and experience by a set of list of the rules [20], [31].

\section{IF 'final exam result' > 65 \\ AND 'attendance' $>70 \%$ \\ THEN 'course grade of the student' is succeed}

In artificial intelligence, rules are most common form which are used for presenting knowledge. A rule is composed of two blocks which are shown in Fig. 1. Rules are described in a "if-then" structure. In this structure, given facts and known information are expressed in "if" block, besides this "then" block is used for action presentation. A rule may present a way of solution for a specific problem. It is relatively easy to create and understand rules [20].

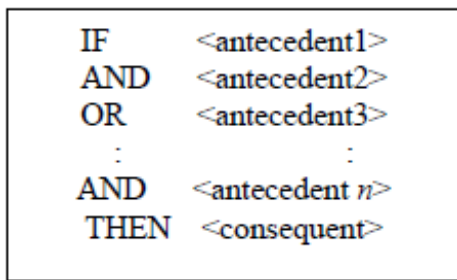

Fig.1. Rules as a knowledge representation technique

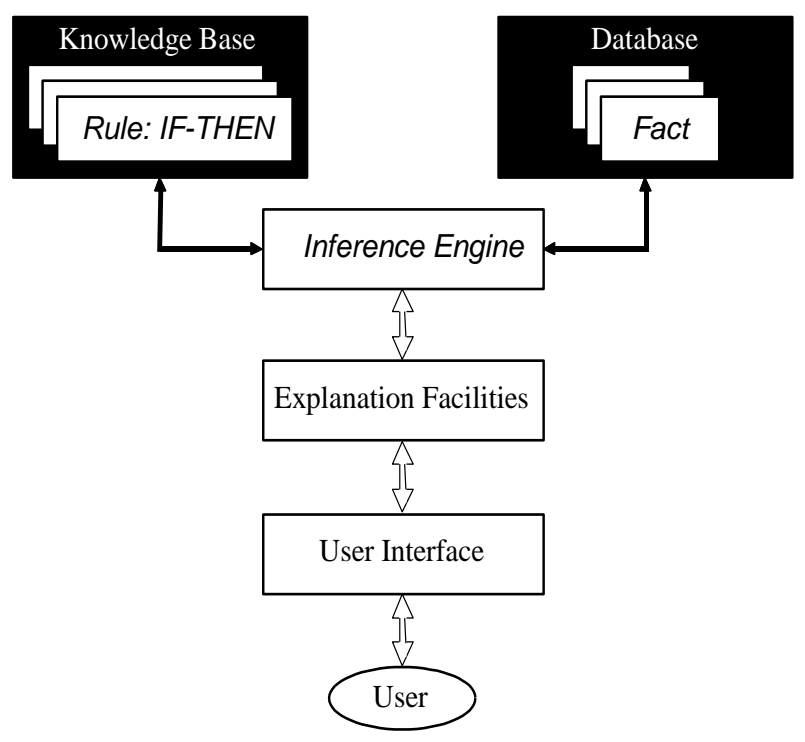

Fig.2. Basic structure of a rule-based expert system [20]. 
In the early 1970s, a model was proposed by Newell and Simon who are from Carnegie-Mellon University. This model constituted basic of modern knowledge base system. System model was built on a concept that solves problems by using of human knowledge. In Fig. 2, system model structure is shown [20].

Knowledge are saved in the way of a set of rules in a rule based expert system. A knowledge base contains useful information to solve a problem in related expert domain. Database consists of a set of facts which are used for matching with "if" block of a rule. Deduction of cause and effect process are obtained by inference engine. Thus, rules and facts connection are established. Explanation ability is a unique feature of expert system. User can ask questions to explanation section about obtained specific results and used facts via user interface. An expert system is developed to run in a specific subject area at level of a human expert. Therefore, high quality performance is most significant property of an expert system. But sometimes expert system may make mistake like a best human expert do [20].

\section{RULE CLASSIFIER AND EXTRACTION ALGORITHMS}

Rule Classifier and Extraction Algorithms are algorithms that work on classifier data set and try to acquire the list of rules that contain and classify all instances. Rule extractions try to summarize the entire data set and put it into a form that is more easily understandable. 3 commonly used rule extractions, part, NNge and prism were preferred in this study.

\section{A. PART}

This algorithm works with the principles of divide and conquer. A partial C4.5 decision tree is obtained and best leaf are transformed as a rule on every iteration. It is seen that, more accurate and complete rules, but less rules quantity are composed rather than $\mathrm{C} 4.5$ decision tree. This method is simple and effective for learning decision lists [32].

\section{B. NNge}

A database is composed with generalized instances which are obtained by nearest neighbor based algorithm.
This method is example of instance-based rule classifier. These database instances are used for generating "if-then" rules by means of Euclidean distance estimation [33], [34].

\section{Prism}

Significant deficiency of Quianlan's ID3 decision tree outputs are hard to understand and not easy to change. Prism method is based on generating rules from training dataset. The reason of using it in expert system is due to support unrelated information. Prism works based on ID3 algorithm and uses different strategies to obtain modular rules list. Thus, prism prevents most problems encountered with decision trees [35].

\section{Methodology of EnRICHING KNOWLEDGE BASE}

Motivation and contribution of this paper is summarizing different methods of the rule extraction from classification data. Multiple rule extraction models are shown and can be used in a novel hybrid way. Thus, readers can use one or more methods which are described in here for the purpose of enriching knowledge base of an expert system. Other related works concentrate on verifying completeness and consistency in a rule based expert system. Our proposed work concentrates on rule extraction from classification data.

Classification data sets are in a format where raw data are held in rows and columns. Row is called either as a record or an instance. The class of the record is indicated by a column which is called class attribute and exists in every instance. Classification data sets, depending on the information inside, contain various knowledge in the related domain. These experiences and knowledge are expressed in terms of rules and used to create the knowledge base of expert systems. As it can be seen in Fig. 3, classification data set is converted into the more valuable list of the rules via rule extraction algorithms. After this stage, to establish an enriched expert system, a semi-auto assessment process to help the knowledge engineer is put into effect and rules are converted into desired form which is more complete and consistent.

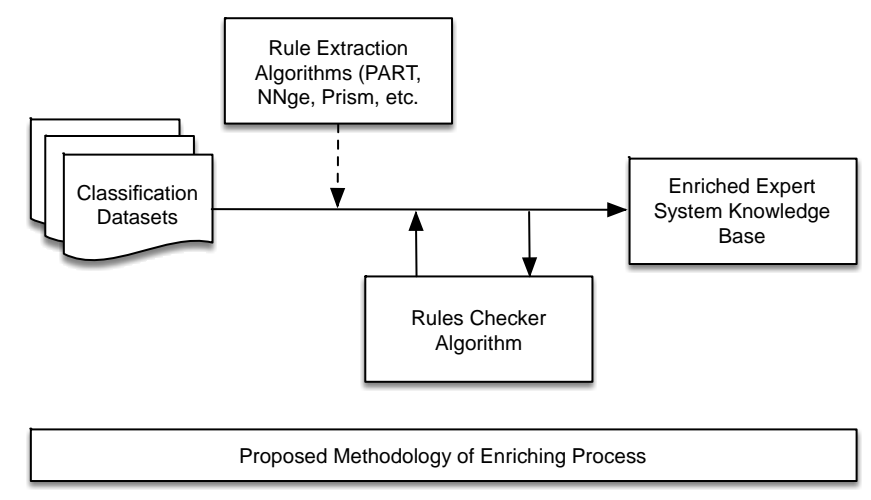

Fig.3. The proposed enriching methodology of knowledge base flow diagram 
In the study, as it can be seen in Table 1, specification of lenses commonly used data set in the UCI machine learning data base [36] is given. Lenses, a database for fitting contact lenses.

Table 1. Used Classification Dataset

\begin{tabular}{|c|c|c|c|}
\hline Dataset & Instances & Attributes & Class \\
\hline lenses & 24 & 4 & 3 \\
\hline
\end{tabular}

The Lenses data set were subjected to Weka software [37] and results were obtained as shown in Table 2 using Part, NNge, Prism algorithms.

Table 2. Extracted Rule Numbers

\begin{tabular}{|c|c|c|c|}
\hline Dataset & Part & NNge & Prism \\
\hline lenses & 4 & 7 & 9 \\
\hline
\end{tabular}

Considering the lenses data set used in this study in detail, the data set is about the suitability of a contact lens for patients. The content and properties of this data set is like the following.

Consists of 3 classes are given as follows:

1: the patient should be fitted with hard contact lenses,

2: the patient should be fitted with soft contact lenses,

3: the patient should not be fitted with contact lenses.

Class distributions are as follows:

1. hard contact lenses: 4

2. soft contact lenses: 5

3. no contact lenses: 15

Data set has 4 attributes and their values are given as follows:

1. age of the patient:

(1) young

(2) pre-presbyopic

(3) presbyopic

Table 3. Lenses Data's Extracted Rules List

\begin{tabular}{|c|c|}
\hline $\begin{array}{c}\text { PART } \\
\text { decision list }\end{array}$ & $\begin{array}{l}\text { tear-prod-rate }=\text { reduced: none }(12.0) \\
\text { astigmatism = no: soft }(6.0 / 1.0) \\
\text { spectacle-prescrip = myope: hard }(3.0) \\
\text { : none }(3.0 / 1.0)\end{array}$ \\
\hline NNge Rules & 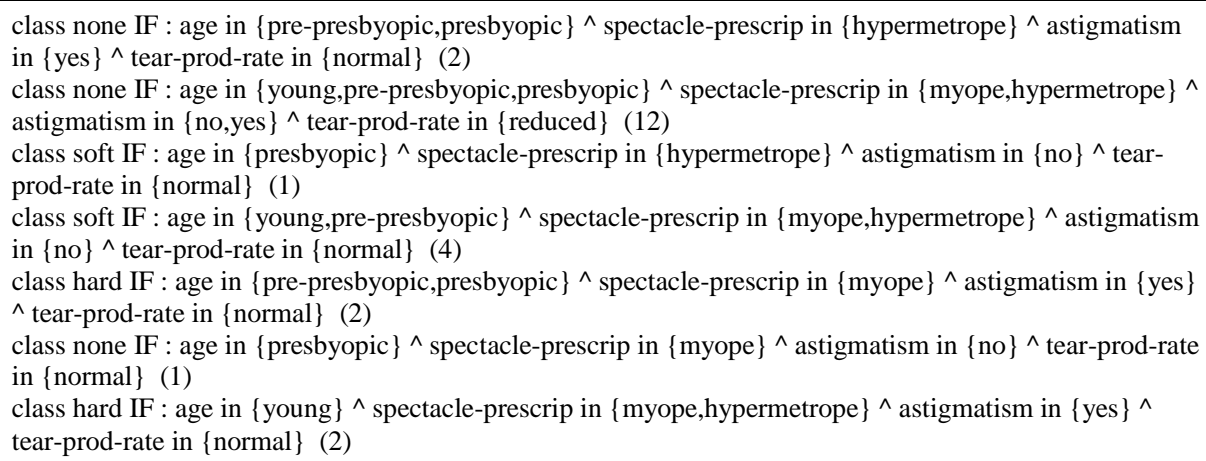 \\
\hline Prism rules & $\begin{array}{l}\text { If astigmatism }=\text { no } \\
\text { and tear-prod-rate }=\text { normal } \\
\text { and spectacle-prescrip }=\text { hypermetrope then soft } \\
\text { If astigmatism }=\text { no } \\
\text { and tear-prod-rate }=\text { normal } \\
\text { and age = young then soft } \\
\text { If age }=\text { pre-presbyopic } \\
\text { and astigmatism }=\text { no } \\
\text { and tear-prod-rate = normal then soft } \\
\text { If astigmatism }=\text { yes } \\
\text { and tear-prod-rate }=\text { normal } \\
\text { and spectacle-prescrip }=\text { myope then hard } \\
\text { If age = young } \\
\text { and astigmatism }=\text { yes } \\
\text { and tear-prod-rate }=\text { normal then hard } \\
\text { If tear-prod-rate }=\text { reduced then none } \\
\text { If age = presbyopic } \\
\text { and tear-prod-rate }=\text { normal } \\
\text { and spectacle-prescrip }=\text { myope } \\
\text { and astigmatism }=\text { no then none } \\
\text { If spectacle-prescrip }=\text { hypermetrope } \\
\text { and astigmatism }=\text { yes } \\
\text { and age }=\text { pre-presbyopic then none } \\
\text { If age }=\text { presbyopic } \\
\text { and spectacle-prescrip }=\text { hypermetrope } \\
\text { and astigmatism }=\text { yes then none }\end{array}$ \\
\hline
\end{tabular}


2. spectacle prescription:

(1) myope

(2) hypermetrope

3. astigmatic:

(1) no

(2) yes

4. tear production rate:

(1) reduced

(2) normal

Algorithm 1. Pseudo Code of Proposed Rule Checker Algorithm

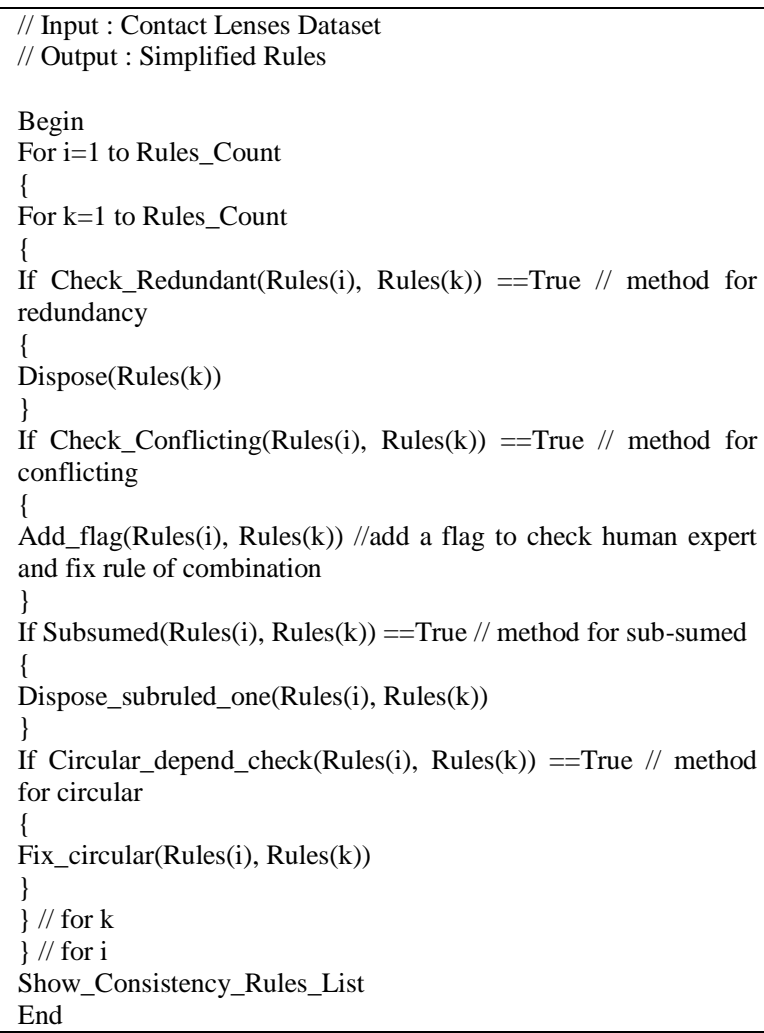

While controlling for redundant rules, similar rules are found. Equals rules in terms of the equality of the antecedent 'if' part and the consequent 'then' part of the rules must be pointed out and redundant rules must be collected under one rule. This is why all the used rule expression and data sets are put into one common format and redundant rules are found.

As it can be seen in Table 3, the first rule of the Part decision list 'tear-prod-rate = reduced: none', the second rule in the NNge list 'class none IF : age in yyoung,prepresbyopic,presbyopic $\} \quad \wedge_{\text {spectacle-prescrip }}$ in $\{\text { myope,hypermetrope }\}^{\wedge}$ astigmatism in $\{$ no,yes $\} \wedge$ tearprod-rate in \{reduced\}', and the rule in Prism 'If tearprod-rate $=$ reduced then none' were found to be the same rules and they need to be expressed as one single rule.

Secondly, conflicting rules are other rules that should be considered. If the 'if' parts of both rules are the same, but the 'then' parts are different, the rules are conflicting rules and a consistency adjustment must be made. There was no such issue in the rules obtained from the lenses data set. If there were rules that conflicted with each other. conflict resolution would have to be implemented. This would require prioritization of the rules.

Another control parameter is about subsumed rules. This issue arises from 'then' statements of one or more rules with unnecessary or additional conditions in the 'if' statements. In this case, the rule in the subset can be dismissed. For example, the third NNge rule 'Class soft IF : age in \{presbyopic\} $\wedge$ spectacle-prescrip in \{hypermetrope $\}^{\wedge}$ astigmatism in $\{\text { no }\}^{\wedge}$ tear-prod-rate in \{normal\}' and the first Prism rule 'If astigmatism $=$ no and tear-prod-rate $=$ normal and spectacle-prescrip $=$ hypermetrope then soft' have the same result 'soft'. However, the results do not change even If the third rule of NNge does not contain the condition 'age in \{presbyopic\}', and this attribute was used unnecessarily here. Extraction of rules this way is an example of subsumed rules control.

Finally, circular rules control is about the condition where the 'then' part of one rules is the 'if' part of another rule. In this case, there may arise an infinite loop among rules and the ability of system to make decision is interrupted [38]. There is no such issue regarding the lenses data set.

In Table 4, after running the rule checker algorithm, list of rules was established for the consistent and complete data base. Table 4 shows the list of all rules obtained by simplification. A total of 20 rules were simplified into 9 rules. All attributes in the list were combined with 'and' statements. The symbol ' $\checkmark$ ', represents 'equal'. The parenthesized values in the rule column show how many of the 24 instances as results of rules are contained by the rules. In the example statement of Rule(Instances), "1 (12)", the number 1 shows the rule number, and the number 12 shows that 12 lenses data set units were covered as a result of running the rule.

\section{CONCLUSION}

This paper outlines a system that runs several rulebased extractors on classification data, combines the output rules to enrich the expert system. In this article, rules were obtained from commonly used classification data sets using rule extraction algorithms. An experiment was conducted on the sample lenses data set by considering whether these results include redundant, conflicting, subsumed and circular rules. Therefore, a method was presented to establish a knowledge base of high content quality. Rule result inference was made and the conflicting rules were eliminated and simplified. The complex structure of the knowledge base was simplified and studies were conducted to simplify the knowledge base in order to create results rapidly.

Expert systems cannot gain new knowledge by themselves, but they need a human with expertise in the relevant domain and a knowledge engineer to input the new information into the system. As disciplines like machine learning develop and the number of data sets increase, classification of data continues in areas such as medicine, space and mechanics etc. It was indicated by 
this study that knowledge-based systems will be enriched by extracting consistent rules from classified data and adding the data on the knowledge base automatically or semi-automatically in control of humans. Experts in a given domain may leave their jobs or be unwilling to provide information, or dangerous and hard working conditions may increase the need for expert systems. In cases where it is difficult to reach an expert, or cases that require expertise in more than one domain, if comprehensive deduction and results are desired, a consistent, complete, and uncomplicated knowledge base can be created systemically using the methods outlined in this study.

As it may be seen from the obtained results, the complex structure of the classification data set where rules are extracted from leads to significantly different results obtained from rule extraction algorithms. Last checks regarding this situation that compromises the system's completeness and stability are left to the domain expert or the knowledge engineer who evaluates the list of rules semi-automatically. For future successful improvement studies on the methods, a more optimized lists of rules can be obtained for artificial intelligence fields like expert systems by ensuring the optimization of genetic algorithms and parameter values of rule algorithms. Therefore, this may be a step to develop a fully automated expert system knowledge base that is more independent and stable.

Table 4. Rule Checker Algorithm's Consistency Rules List

\begin{tabular}{|c|c|c|c|c|c|c|c|c|c|c|c|c|}
\hline \multirow{2}{*}{$\begin{array}{c}\text { Rule } \\
\text { (Instances) }\end{array}$} & \multicolumn{3}{|c|}{ Age } & \multicolumn{2}{|c|}{ Spectacle } & \multicolumn{2}{|c|}{ Astig. } & \multicolumn{2}{|c|}{ Tear } & \multicolumn{3}{|c|}{ Class } \\
\hline & Young & Pre-Pres. & Pre. & Мyо. & Hyper. & Yes & No & Reduced & Normal & Hard & Soft & None \\
\hline $1(12)$ & & & & & & & & $\checkmark$ & & & & $\checkmark$ \\
\hline $2(3)$ & & & & $\checkmark$ & & $\checkmark$ & & & $\checkmark$ & $\checkmark$ & & \\
\hline $3(2)$ & & $\checkmark$ & & & $\checkmark$ & $\checkmark$ & & & & & & $\checkmark$ \\
\hline $4(2)$ & & & $\checkmark$ & & $\checkmark$ & $\checkmark$ & & & & & & $\checkmark$ \\
\hline $5(2)$ & $\checkmark$ & & & & & & $\checkmark$ & & $\checkmark$ & & $\checkmark$ & \\
\hline $6(2)$ & & $\checkmark$ & & & & & $\checkmark$ & & $\checkmark$ & & $\checkmark$ & \\
\hline $7(1)$ & & & $\checkmark$ & $\checkmark$ & & & $\checkmark$ & & $\checkmark$ & & & $\checkmark$ \\
\hline $8(2)$ & $\checkmark$ & & & & & $\checkmark$ & & & $\checkmark$ & $\checkmark$ & & \\
\hline $9(3)$ & & & & & $\checkmark$ & & $\checkmark$ & & $\checkmark$ & & $\checkmark$ & \\
\hline
\end{tabular}

\section{ACKNOWLEDGMENT}

We would like to thank the anonymous reviewers for their comments which significantly improved the exposition.

\section{REFERENCES}

[1] Onisko A, Lucas P, Druzdzel MJ. Comparison of rulebased and Bayesian network approaches in medical diagnostic systems. Lect Notes Artif Int. 2001;2101:28392.

[2] Eghbali S, Ayatollahi S, Boozarjomehry RB. New expert system for enhanced oil recovery screening in nonfractured oil reservoirs. Fuzzy Set Syst. 2016;293:80-94.

[3] Mehdi KHOSRAVI, Mohammad YAZDANSHENAS, Mohammad Hossein NEMATI, Design of an Expert System for Diagnosis of Thyroid Cancer, Cumhuriyet University Faculty of Science Science Journal (CSJ), Vol. 36, No: 3 Special Issue (2015) ISSN: 1300-1949

[4] Nascimento DA, Anunciacao RM, Arnhold A, Ferraz AC, dos Santos A, Zanuncio JC. Expert system for identification of economically important insect pests in commercial teak plantations. Comput Electron Agr. 2016;121:368-73.

[5] Ramirez G, Valenzuela MA, Lorenz RD. Expert System for the Detection of Condensate Accumulation Inside Dryer Cylinders During Section Starting. Ieee T Ind Appl. 2015;51(2):1427-37.

[6] Keles A. Expert Doctor Verdis: Integrated medical expert system. Turk J Electr Eng Co. 2014;22(4):1032-43.

[7] Liao SH. Expert system methodologies and applications a decade review from 1995 to 2004. Expert Syst Appl. 2005;28(1):93-103.

[8] Sai TK, Reddy KA. New Rules Generation From Measurement Data Using an Expert System in a Power Station. Ieee T Power Deliver. 2015;30(1):167-73.

[9] Ikram A, Qamar U. Developing an expert system based on association rules and predicate logic for earthquake prediction. Knowl-Based Syst. 2015;75:87-103.

[10] Bashir S, Qamar U, Khan FH, Javed MY. MV5: A Clinical Decision Support Framework for Heart Disease Prediction Using Majority Vote Based Classifier Ensemble. Arab J Sci Eng. 2014;39(11):7771-83.

[11] Balouchi B, Nikoo MR, Adamowski J. Development of 
expert systems for the prediction of scour depth under live-bed conditions at river confluences: Application of different types of ANNs and the M5P model tree. Appl Soft Comput. 2015;34:51-9.

[12] K.Mani, R.Akila,"Enhancing the Performance in Generating Association Rules using Singleton Apriori", International Journal of Information Technology and Computer Science(IJITCS), Vol.9, No.1, pp.58-64, 2017. DOI: 10.5815/ijitcs.2017.01.07

[13] Coussement K, Benoit DF, Antioco M. A Bayesian approach for incorporating expert opinions into decision support systems: A case study of online consumersatisfaction detection. Decis Support Syst. 2015;79:24-32.

[14] Dongjin Park, Taeho Cho,"A Fuzzy Rule-based Key ReDistribution Decision Scheme of Dynamic Filtering for Energy Saving in Wireless Sensor Networks", International Journal of Information Technology and Computer Science(IJITCS), Vol.9, No.4, pp.1-8, 2017. DOI: $10.5815 /$ ijitcs.2017.04.01

[15] Yin JT, Chen DW, Li LX. Intelligent Train Operation Algorithms for Subway by Expert System and Reinforcement Learning. Ieee $\mathrm{T}$ Intell Transp. 2014;15(6):2561-71.

[16] Sarkar S, Sharma T, Baral A, Chatterjee B, Dey D, Chakravorti S. An Expert System Approach for Transformer Insulation Diagnosis combining Conventional Diagnostic Tests and PDC, RVM Data. Ieee T Dielect El In. 2014;21(2):882-91.

[17] Ighoyota Ben Ajenaghughrure, P. Sujatha, Maureen I. Akazue, "Fuzzy Based Multi-Fever Symptom Classifier Diagnosis Model", International Journal of Information Technology and Computer Science(IJITCS), Vol.9, No.10, pp.13-28, 2017. DOI: 10.5815/ijitcs.2017.10.02

[18] Sahar Abdalla Elmubarak, Adil Yousif, Mohammed Bakri Bashir,"Performance based Ranking Model for Cloud SaaS Services", International Journal of Information Technology and Computer Science(IJITCS), Vol.9, No.1, pp.65-71, 2017. DOI: 10.5815/ijitcs.2017.01.08

[19] Omid M. Design of an expert system for sorting pistachio nuts through decision tree and fuzzy logic classifier. Expert Syst Appl. 2011;38(4):4339-47.

[20] M. Negnevitsky, Artificial intelligence: a guide to intelligent systems, Pearson Education, 2005

[21] Chang LL, Zhou ZJ, You Y, Yang LH, Zhou ZG. Belief rule based expert system for classification problems with new rule activation and weight calculation procedures. Inform Sciences. 2016;336:75-91.

[22] D'Haen J, Van den Poel D, Thorleuchter D, Benoit DF. Integrating expert knowledge and multilingual web crawling data in a lead qualification system. Decis Support Syst. 2016;82:69-78.

[23] Yin JT, Chen DW, Li YD. Smart train operation algorithms based on expert knowledge and ensemble CART for the electric locomotive. Knowl-Based Syst. 2016;92:78-91.

[24] Ho HF, Li ST. Using mutually validated memories of experts for case-based knowledge systems. Knowl-Based Syst. 2015;86:102-15.

[25] Laasri EHA, Akhouayri ES, Agliz D, Zonta D, Atmani A. A fuzzy expert system for automatic seismic signal classification. Expert Syst Appl. 2015;42(3):1013-27.

[26] Seok J, Kasa-Vubu J, DiPietro M, Girard A. Expert system for automated bone age determination. Expert Syst Appl. 2016;50:75-88.

[27] Ghanbari A, Kazemi SMR, Mehmanpazir F, Nakhostin MM. A Cooperative Ant Colony Optimization-Genetic Algorithm approach for construction of energy demand forecasting knowledge-based expert systems. KnowlBased Syst. 2013;39:194-206.

[28] Murianaa Cinzia, Piazzaa Tommaso, Vizzinib Giovanni, An expert system for financial performance assessment of health care structures based on fuzzy sets and KPIs. Knowl-Based Syst. 2016:97:1-10

[29] Lee KC, Lee S. A causal knowledge-based expert system for planning an Internet-based stock trading system. Expert Syst Appl. 2012;39(10):8626-35.

[30] Xavier D, Crespo B, Fuentes-Fernandez R. A rule-based expert system for inferring functional annotation. Appl Soft Comput. 2015;35:373-85.

[31] Zhou Zude, Manufacturing Intelligence for Industrial Engineering: Methods for System Self-Organization, Learning, and Adaptation: Methods for System SelfOrganization, Learning, and Adaptation, IGI Global, 2010

[32] Eibe Frank, Ian H. Witten: Generating Accurate Rule Sets Without Global Optimization. In: Fifteenth International Conference on Machine Learning, 144-151, 1998.

[33] Brent Martin (1995). Instance-Based learning: Nearest Neighbor With Generalization. Hamilton, New Zealand.

[34] Sylvain Roy (2002). Nearest Neighbor With Generalization. Christchurch, New Zealand

[35] Cendrowska J. Prism - an Algorithm for Inducing Modular Rules. Int J Man Mach Stud. 1987;27(4):349-70.

[36] Lichman, M. (2013). UCI Machine Learning Repository [http://archive.ics.uci.edu/ml]. Irvine, CA: University of California, School of Information and Computer Science.]

[37] Mark Hall, Eibe Frank, Geoffrey Holmes, Bernhard Pfahringer, Peter Reutemann, Ian H. Witten (2009); The WEKA Data Mining Software: An Update; SIGKDD Explorations, Volume 11, Issue 1.

[38] T. A. Nguyen , W. A. Perkins , T. J. Laffey and D. Pecora, "Checking an expert sytems knowledge base for consistency and completeness", Proc. IJCAI-85, pp. 375378, 1985

[39] M. Suwa, A.C. Scott, E.H. Shortliffe, An approach to verifying completeness and consistency in a rule-based expert system, AI Mag., 3 (3) (1982), pp. 16-21

\section{Authors' Profiles}

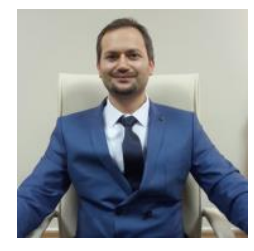

İbrahim Berkan Aydilek received the $\mathrm{PhD}$ degree in computer engineering from Selçuk University. $\mathrm{He}$ is an assistant professor of computer science at Harran University, Sanlıurfa, Turkey. His research interests include machine learning, data mining and artificial intelligence.

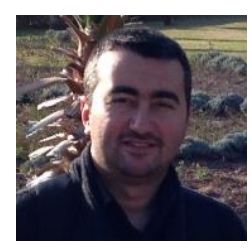

Abdülkadir Gümüşçü is assistant professor at Harran University Electrical and Electronics Engineering Department. $\mathrm{He}$ got Ph.D degree in Electrical and Electronics Engineering from Harran University, Şanlıurfa, Turkey. His main research interests are image processing and

machine learning.

How to cite this paeper: İbrahim Berkan Aydilek, Abdülkadir Gümüşçü, "Designing a Rule Based Expert Systems for Contact Lenses Patients", International Journal of Information 
Technology and Computer Science(IJITCS), Vol.10, No.3, pp.18-26, 2018. DOI: 10.5815/ijitcs.2018.03.03 